Artículo original

\title{
INFLUENCIA DE LA TEMPERATURA Y VELOCIDAD DEL FLUJO DE AIRE EN LAS CARACTERÍSTICAS FÍSICA Y SENSORIALES DEL TOMATE (LYCOPERSICUM ESCULENTUM) DESHIDRATADO EN SECADOR DE BANDEJAS "INFLUENCE OF TEMPERATURE AND AIR FLOW VELOCITY ON THE PHYSICAL AND SENSORY CHARACTERISTICS OF TOMATO (LYCOPERSICUM ESCULENTUM) DEHYDRATED IN TRAY DRYER"
}

Roberto Antonio Palacios Flores ${ }^{1}$ Dr. Raul Cartagena Cutipa ${ }^{1,2}$

Información del artículo: Recibido: 18/06/2019. Aceptado: 22/11/2019

${ }^{1}$ Ingeniero Agroindustrial

${ }^{2}$ Docente Facultad de Ingeniería en Universidad Privada de Tacna 
Vol. $1, \mathrm{~N}^{\circ} 1$.

Julio - Diciembre del 2019.

\section{Resumen}

El principal objetivo fue evaluar la influencia de la temperatura y velocidad del flujo de aire en el tiempo de deshidratación. Se caracterizó la materia prima basado en las normas de la AOAC, reportando $94,5 \%$ de humedad, $3,94 \mathrm{pH} ; 4,5^{\circ}$ Brix y 0,93\% de acidez titulable expresada en ácido cítrico. El proceso de deshidratación se realizó con temperaturas de 55,65 y $75{ }^{\circ} \mathrm{C}$ a velocidades de 0,$6 ; 0,9$ y $1,2 \mathrm{~m} / \mathrm{s}$, las rebanadas de tomate de $5 \pm 0,2 \mathrm{~mm}$ de espesor; se sometieron a un pre-tratamiento en una solución de metabisulfito de sodio al 0,2 \% + cloruro de calcio al $1 \%$, donde a la mayor temperatura el proceso de deshidratación culminó en $7 \mathrm{~h}$, mientras que a la menor temperatura deshidrato en $13 \mathrm{~h}$. El porcentaje de encogimiento de las rebanadas de tomate estuvo entre $18,02-28,81$. Las evaluaciones sensoriales realizadas con pruebas de aceptación por panelistas no entrenados, resultó en diferencias estadísticamente significativas en las características de color, textura y apariencia más no así en la característica color de las muestras deshidratadas a un $95 \%$ de confianza.

Palabras clave: deshidratación, tomate, encogimiento, propiedades físico- químicas.

\section{Abstract}

The main objective was to evaluate the influence of the temperature and velocity of air in the time of dehydration. The raw material was characterized based on the norms of the AOAC, reporting $94,5 \%$ of humidity, $3,94 \mathrm{pH} ; 4,5^{\circ}$ Brix and $0,93 \%$ titratable acidity expressed in citric acid. The dehydration process was carried out with temperatures of 55,65 and $75{ }^{\circ} \mathrm{C}$ at speeds of 0,$6 ; 0,9$ and $1,2 \mathrm{~m} / \mathrm{s}$, the tomato slices of $5 \pm 0,2 \mathrm{~mm}$ thick; They underwent a pre-treatment in a solution of sodium metabisulfite to $0,2 \%+$ calcium chloride at $1 \%$, where at the highest temperature the dehydration process culminated in $7 \mathrm{~h}$, while at the lowest temperature dehydrate in $13 \mathrm{~h}$. The percentage of shrinkage of tomato slices was between $18,02-28,81$. Sensory evaluations carried out with evidence of acceptance by untrained panelists resulted in statistically significant differences in odor, texture and appearance characteristics but not in the characteristic color of the dehydrated samples at $95 \%$ confidence.

Key words: dehydration, tomato, shrinkage, physico-chemical properties.

\section{INTRODUCCIÓN}

El tomate es originario de América tropical, probablemente en México o en Perú. Fue llevado a Europa a principios del siglo XVI y más tarde en el siglo XVIII a América del Norte y desde estas áreas se extendió al resto del mundo (Jenkins, 1948). El tomate con nombre científico "Lycopersicum esculentum" hoy en día es indispensable en la dieta diaria. Tiene un difícil manejo post cosecha, lo que ocasiona pérdidas importantes de producto durante su almacenamiento y comercialización, razón importante para considerar métodos de conservación, como la deshidratación y así extender su vida útil (Moreno, Sierra, y Díaz- 
Moreno, 2014). Mediante la deshidratación se logra reducir el contenido de humedad, se reduce su actividad de agua (aw), inhibiendo al máximo el desarrollo de microorganismos y la velocidad de las reacciones químicas y enzimáticas. Las condiciones favorables del clima y las posibilidades que ofrecen los recursos naturales en el Perú, permiten la obtención de productos de óptima calidad, tal es el caso del tomate, el cual es un producto con bastante demanda en la dieta diaria, sin embargo, contiene un alto nivel de agua mayor al $90 \%$ por lo que lo hace muy vulnerable al deterioro y por consecuencia tiene un periodo corto de vida útil. El tomate al ser un fruto altamente perecedero, es uno de los productos a los que se le debería prestar gran atención por sus múltiples beneficios, tanto nutricionales como económicos; con la deshidratación se reducen gastos en almacenamiento y transporte, por lo cual es necesario estudiar la influencia de las principales variables en el proceso de deshidratación como son la temperatura $\left(\mathrm{T}^{\circ} \mathrm{C}\right)$ y velocidad del aire (vfa). Moreno, Sierra, y Díaz-Moreno (2014) estudiaron los parámetros fisicoquímicos, microbiológicos y sensoriales. Obtuvo que el color y la textura son los parámetros más importantes en cuanto a la calidad sensorial del tomate. Moreno y Díaz (s.f.) evaluaron la influencia de tres temperaturas $\left(50,60\right.$ y $\left.70^{\circ} \mathrm{C}\right)$ en el color y la textura, encontrando que a $70^{\circ} \mathrm{C}$ la coloración roja se ve disminuida haciendo que el color en general cambie y que la temperatura de $60^{\circ} \mathrm{C}$ es la más adecuada texturalmente ya las muestras tienen más fracturabilidad que las muestras deshidratadas a 50 y $70^{\circ} \mathrm{C}$ y un buen balance de masticabilidad y dureza. Monsalve y Machado (2007) realizaron una evaluación de la deshidratación del tomate por dos equipos de secado, la estufa al aire (EA) y el secador de bandejas (SB). La deshidratación se pudo realizar sin ningún problema. Entre los dos procesos, el mejor fue el de (SB) $(p<0,05)$. Gómez (2009) realizó el deshidratado de tomate variedad Saladette en un secador de charolas. Observaron que la rotación de charolas tiene una contribución sobre el color (a*), licopeno y ácido ascórbico. Doymaz (2006 evaluó la capacidad de rehidratación. Los tomates pre-tratados con solución alcalina de oleato de etilo deshidrataron más rápido que las muestras sin tratar. Maldonado, Amau y Bertuzzi (2009) evaluaron el efecto del pre- tratamiento y temperatura en la difusión de agua durante la rehidratación en mangos deshidratados. Demostraron que la rehidratación puede ser interpretada por la difusión de Fickian y que el coeficiente de difusión de agua eficaz es mayor a $40^{\circ} \mathrm{C}$ que a 25 o $60^{\circ} \mathrm{C}$.

\section{OBJETIVOS}

- Evaluar el efecto de la temperatura y velocidad del flujo de aire en el tiempo de deshidratación.

- Evaluar las características físicas y sensoriales del tomate deshidratado.

\section{METODOLOGÍA}

La investigación fue de tipo descriptivo y de nivel experimental. Se realizó un diseño factorial multinivel de 9 tratamientos para el proceso de deshidratación de las rebanadas de tomate y para el análisis posterior de los resultados obtenidos. El estudio se realizó con tomates variedad Río grande mejorado comprados en un mercado local, provenientes de la región Tacna. Los tomates tuvieron una dureza que varía entre 4,50 y 5,68 kg/ y entre un 4,6 y 4,8 de índice de madurez para realizar la deshidratación, presentaron una cáscara relativamente gruesa, sin rajaduras ni grietas, la sensación al tacto debe sentirse duro, ante una suave presión y el color de la cáscara debe ser $100 \%$ roja. A continuación, se les realizó un lavado sumergiendo los tomates en agua clorada, se cortaron los tomates en rebanadas de entre 2 a $5 \mathrm{~mm}$ de grosor. 
Se sumergirán en una solución de metabisulfito de sodio al 0,2 \% y cloruro de calcio al $1 \%$ por 10 minutos a temperatura ambiente. Se distribuyeron en 5 bandejas, de $31 \times 42 \mathrm{~cm}, 9$ rebanadas por bandeja. Las bandejas fueron dispuestas para la deshidratación en el secador. En esta etapa se controlaron las variables temperatura $\left(T^{\circ} \mathrm{C}\right)$ y velocidad del flujo de aire (vfa). Las rebanadas deshidratadas se envasaron en bolsas plásticas impermeables. Se almacenaron en un lugar fresco y seco, hasta cuando se realice las evaluaciones sensoriales.

\section{Técnicas}

\section{En la materia prima:}

a) Preparación de la muestra. CODEX STAN 247-2005

b) Medición del pH. Determinación por potenciometría: Método ISO 1842:1991

c) Determinación de la acidez titulable. Determinación por colorimetría: Método 942.15 (37.1.37) del AOAC (1996)

d) Determinación de los sólidos solubles. Determinación indirecta por refractometría: Método 983.17 del AOAC, 1996

e) Determinación del Índice de Madurez (IM)

f) Determinación de la humedad. Determinación por gravimetría: Método 934.06 (37.1.10) del AOAC, 1996

\section{En los tratamientos:}

a) Determinación de la humedad. Determinación por gravimetría: Método 934.06 (37.1.10) del AOAC, 1996

\section{RESULTADOS}

En la tabla 1 se muestran los resultados de la caracterización físico- química del tomate variedad Río Grande mejorado, donde el Índice de Maduración estuvo entre 4,6 a 4,8; la determinación de humedad reportó un $94,5 \%$ en base húmeda. En cuanto a pH se encontró un valor de 3,94 unidades. Los sólidos solubles en el tomate se tuvó $4,5^{\circ}$ Brix en promedio. La acidez estuvo en 0,93\% expresado en ácido cítrico.

Tabla 1. Resultados de la caracterización físico-química de la materia prima

\begin{tabular}{l|l}
\hline ITEM & RESULTADO \\
\hline IM & $4,6-4,8$ \\
\hline Humedad & $94,5 \%$ \\
\hline $\mathrm{pH}$ & 3,94 \\
\hline Sólidos solubles & $4,5^{\circ}$ Brix \\
\hline Acidez & $0,93 \%$ ácido cítrico \\
\hline
\end{tabular}

En la tabla 2 se muestra los resultados del análisis de varianza donde los factores temperatura y velocidad del flujo de aire influyen significativamente en el tiempo de deshidratación de los tomates en rebanadas con un $95 \%$ de confianza, ya que ambos factores presentan un valor menor a 0,05 de significancia.

Tabla 2. Análisis de varianza para los factores temperatura y velocidad en el tiempo de deshidratación.

\begin{tabular}{cccccc}
\hline Origen & Suma de Cuadrados & gl & Media Cuadrática & F & Sig. \\
\hline A:Temperatura & 20.2222 & 2 & 10.1111 & 7.91 & 0.0407
\end{tabular}




\begin{tabular}{llllll} 
B:Velocidad del aire & 20.2222 & 2 & 10.1111 & 7.91 & 0.0407 \\
RESIDUOS & 5.11111 & 4 & 1.27778 & & \\
\hline
\end{tabular}

En la tabla 3 se observa la prueba de rango múltiple entre la temperatura y el tiempo de deshidratación observamos dos grupos, donde las temperaturas de $55^{\circ} \mathrm{C}$ y $75^{\circ} \mathrm{C}$ se muestran diferencias estadísticamente significativas referentes al tiempo de deshidratación con un nivel del $95 \%$ de confianza. En la tabla 4 de la prueba de rango múltiple entre la velocidad y el tiempo de deshidratación observamos dos grupos, donde la velocidad de $0,6 \mathrm{~m} / \mathrm{s}$ con 1,2 m/s y $0,9 \mathrm{~m} / \mathrm{s}$ muestra diferencias estadísticamente significativas referentes al tiempo de deshidratación con un nivel del $95 \%$ de confianza.

Tabla 3. Prueba de rango múltiple de Duncan $(P=0.05)$ para la temperatura en el tiempo de deshidratación.

\begin{tabular}{lccccc}
\hline Orden & Tratamiento $\left({ }^{\circ} \mathrm{C}\right)$ & Promedio $(\mathrm{h})$ & \multicolumn{2}{c}{ Significancia $\alpha=0,05$} & \\
\hline 1 & 55 & 10,0 & & $\mathrm{a}$ & \\
2 & 65 & 8,3 & $\mathrm{a}$ & & $\mathrm{b}$ \\
3 & 75 & 6,3 & & $\mathrm{~b}$ & \\
\hline
\end{tabular}

Tabla 4.

Prueba de rango múltiple de Duncan $(\mathrm{P}=0.05)$ para la velocidad en el tiempo de deshidratación.

\begin{tabular}{lccc}
\hline Orden & Tratamiento $(\mathrm{m} / \mathrm{s})$ & Promedio $(\mathrm{h})$ & Significancia $\alpha=0,05$ \\
\hline 1 & 0,6 & 10,3 & $\mathrm{a}$ \\
2 & 1,2 & 7,3 & $\mathrm{~b}$ \\
3 & 0,9 & 7,0 & $\mathrm{~b}$ \\
\hline
\end{tabular}

En la tabla 5 se muestran los resultados de la medición de las superficies de las rebanadas de tomate, realizadas antes y después de cada tratamiento de deshidratación, también se midió el espesor y se calculó el porcentaje de encogimiento por cada tratamiento. El porcentaje de encogimiento estuvo entre un $18,02-28,81 \%$. El tratamiento T1 tuvo el menor porcentaje de encogimiento y por el contrario el tratamiento T9 tuvo el mayor porcentaje. Estos resultados se deben en T9; la alta temperatura $\left(75^{\circ} \mathrm{C}\right)$ y velocidad $(1,2 \mathrm{~m} / \mathrm{s})$ y por el contrario en $\mathrm{T} 1\left(55^{\circ} \mathrm{C}\right.$ y $\left.0,6 \mathrm{~m} / \mathrm{s}\right)$ se produjeron estos valores de encogimiento debido a factores que influyen en el tamaño y forma de las rebanadas de tomate siendo la temperatura y velocidad de flujo de aire, también otro factor es el alto contenido de humedad 94,2\%.

Tabla 5. Superficie y espesor de las rebanadas de tomate

\begin{tabular}{llllll}
\hline \multirow{2}{*}{ Tratamiento } & \multicolumn{2}{l}{ Superficie $\left(\mathrm{cm}^{2}{ }^{2}{ }_{-}\right.$} & \multicolumn{2}{l}{ Espesor $(\mathrm{cm})$} & Encogimiento (\%) \\
& Inicial & Final & Inicial & Final & \\
\hline T1 & 6,05 & 4,96 & 0,40 & 0,20 & 18.02 \\
T2 & 6,10 & 4,89 & 0,45 & 0,20 & 19.84 \\
T3 & 5,90 & 4,72 & 0,42 & 0,20 & 20.00 \\
T4 & 6,00 & 4,59 & 0,38 & 0,20 & 23.50 \\
T5 & 6,10 & 4,60 & 0,48 & 0,20 & 24.59 \\
T6 & 5,90 & 4,45 & 0,43 & 0,20 & 24.58 \\
T7 & 5,80 & 4,40 & 0,44 & 0,20 & 24.14 \\
T8 & 6,20 & 4,43 & 0,49 & 0,20 & 28.55 \\
T9 & 5,90 & 4,20 & 0,44 & 0,20 & 28.81 \\
\hline
\end{tabular}


La temperatura no tiene significancia ( $p$ : 0.06) pero la velocidad del aire influye significativamente en el encogimiento ( $p: 0.00$ ). Todas las velocidades 0,$6 ; 0,9$ y $1,2 \mathrm{~m} / \mathrm{s}$ tienen diferencias estadísticamente significativas $(p=0.05)$ en el encogimiento de las rebanadas de tomate a un nivel de $95 \%$ de confianza.

Para evaluar la influencia de la temperatura y velocidad del aire en las características sensoriales del tomate deshidratado, se realizaron las pruebas con el fin de determinar el grado de satisfacción. Los parámetros que se midieron fueron: Color, olor, textura y apariencia del tomate deshidratado.

En la tabla 6 observamos no existe una diferencia significativa entre las muestras evaluadas $(p=0,05)$. Para el olor se encontraron diferencias significativas entre la muestra T7 y difieren de las muestras T1, T9, T6, T8, T2 y T5. En la textura se encontraron diferencias significativas entre las muestras T1 y T6, donde estas difieren ambas con las muestran T8 y T3. En la apariencia se encontraron diferencias significativas entre las muestras T1, T6, T5, T8 y T3.

Tabla 6. Resultados de Media y prueba de rango múltiple de Duncan $(p=0,05)$

\begin{tabular}{|c|c|c|c|c|c|c|c|c|}
\hline \multicolumn{9}{|c|}{ COLOR } \\
\hline $\mathrm{T} 1$ & T4 & $\mathrm{T} 2$ & T5 & T6 & T9 & T8 & $\mathrm{T7}$ & T3 \\
\hline $3,5 a$ & $3,3 a$ & $3,3 a$ & $3,2 \mathrm{a}$ & $3,2 a$ & $3,1 a$ & $3,1 a$ & $3,0 a$ & $3,0 a$ \\
\hline \multicolumn{9}{|c|}{ OLOR } \\
\hline$T 7$ & T4 & T3 & T1 & T9 & T8 & T2 & T5 & T6 \\
\hline $3,6 a$ & $3,3 a b$ & $3,3 a b$ & $3,0 b$ & $3,0 b$ & $3,0 b$ & $2,9 b$ & $2,8 \mathrm{~b}$ & $2,7 b$ \\
\hline \multicolumn{9}{|c|}{ TEXTURA } \\
\hline$T 6$ & T1 & T7 & T9 & T5 & $\mathrm{T} 2$ & T4 & T3 & T8 \\
\hline $3,2 a$ & $3,2 a$ & $3,0 a b$ & $2,9 a b$ & $2,8 a b$ & $2,8 a b$ & $2,7 a b$ & $2,5 b$ & $2,4 b$ \\
\hline \multicolumn{9}{|c|}{ APARIENCIA } \\
\hline $\mathrm{T1}$ & T6 & T5 & $\mathrm{T7}$ & T9 & T4 & T2 & T8 & T3 \\
\hline $3,3 a$ & $3,2 \mathrm{ab}$ & $3,0 a b$ & $2,7 a b c$ & $2,7 a b c$ & $2,7 a b c$ & $2,7 a b c$ & $2,6 b c$ & $2,2 \mathrm{c}$ \\
\hline
\end{tabular}

Tabla 7. Medias de los tratamientos luego de la evaluación sensorial

\begin{tabular}{cc}
\hline TRATAMIENTO & MEDIA \\
\hline T1 $\left(55^{\circ} \mathrm{C} ; 0,6 \mathrm{~m} / \mathrm{s}\right)$ & 3,25 puntos \\
T7 $\left(55^{\circ} \mathrm{C} ; 1,2 \mathrm{~m} / \mathrm{s}\right)$ & 3,08 puntos \\
T6 $\left(75^{\circ} \mathrm{C} ; 0,9 \mathrm{~m} / \mathrm{s}\right)$ & 3,08 puntos \\
T4 $\left(55^{\circ} \mathrm{C} ; 0,9 \mathrm{~m} / \mathrm{s}\right)$ & 3,00 puntos \\
T5 $\left(65^{\circ} \mathrm{C} ; 0,9 \mathrm{~m} / \mathrm{s}\right)$ & 2,95 puntos \\
T2 $\left(65^{\circ} \mathrm{C} ; 0,6 \mathrm{~m} / \mathrm{s}\right)$ & 2,93 puntos \\
T9 $\left(75^{\circ} \mathrm{C} ; 1,2 \mathrm{~m} / \mathrm{s}\right)$ & 2,93 puntos \\
T8 $\left(65^{\circ} \mathrm{C} ; 1,2 \mathrm{~m} / \mathrm{s}\right)$ & 2,78 puntos \\
T3 $\left(75^{\circ} \mathrm{C} ; 0,6 \mathrm{~m} / \mathrm{s}\right)$ & 2,75 puntos \\
\hline
\end{tabular}

En conclusión, las primeras muestras con el mayor puntaje global fueron de los tratamientos: $\mathrm{T} 1\left(55^{\circ} \mathrm{C} ; 0,6 \mathrm{~m} / \mathrm{s}\right)$ con 3,25 puntos y $\mathrm{T} 7\left(55^{\circ} \mathrm{C} ; 1,2 \mathrm{~m} / \mathrm{s}\right)$ con 3,08 puntos mientras que las 
últimas: T8 $\left(65{ }^{\circ} \mathrm{C} ; 1,2 \mathrm{~m} / \mathrm{s}\right)$ y $\mathrm{T} 3\left(75{ }^{\circ} \mathrm{C} ; 0,6 \mathrm{~m} / \mathrm{s}\right)$ obtuvieron 2,78 y 2,75 puntos respectivamente. (Tabla 7 )

\section{DISCUSIÓN}

Se han mostrado los resultados de la caracterización físico- química de la materia prima. La determinación de humedad en tomate reportó un $94,5 \%$ en base húmeda, este valor es similar a lo hallado por Monsalve \& Machado (2007) y por Doymaz (2006) de 94,4 \% y 94,5\% respectivamente. La humedad es el contenido de agua en $100 \mathrm{~g}$ de muestra (Moreno \& Díaz, s.f.). En cuanto a pH se encontró un valor de 3,94 unidades siendo similar a 4,36 hallado por Gómez \& Camelo (2002), el pH se calcula por la concentración de iones de hidrógeno, un factor que controla la regulación de muchas reacciones químicas, bioquímicas y microbiológicas. Los sólidos solubles constituyen un parámetro empleado comúnmente en el análisis de alimentos y bebidas, en especial en frutas, se definen como todas aquellas sustancias que normalmente se presentan en estado sólido bajo condiciones ambientales pero que en ciertas circunstancias pasan a formar parte de una solución, azúcares y sales, en el tomate se tuvo $4,5^{\circ} \mathrm{Brix}$ en promedio, mientras que Panzo (2012) reportó $5,46^{\circ}$ Brix y Gómez \& Camelo (2002) encontraron un valor de $4,23^{\circ}$ Brix estos valores resultan diferentes debido a diversos factores como: Estado de madurez de los tomates, la estación en que fueron cosechados, la ubicación geográfica, el clima, la composición del suelo, entre otros. La acidez estuvo en 0,93 \% expresado en ácido cítrico mientras que Gómez \& Camelo (2002) hallaron un valor de $0,45 \%$ se encuentran diferencias debido a que los tomates en estudio estaban en un estado de madurez más avanzada. Los ácidos son sustancias biológicamente activas que contribuyen en mayor proporción a la capacidad antioxidante de las hortalizas confiriéndoles color, aroma y sabor no considerados como nutrientes esenciales pero su efecto en la salud es positivo.

En la deshidratación de las muestras de tomate se usó el pretratamiento de una solución de cloruro de calcio + metabisulfito de potasio, esto ayuda a una rápida evaporación de la superficie mojada de las rebanadas de tomate y de esta manera el agua menos ligada se remueve constantemente por difusión rápida desde el interior, así también por la composición molecular del cloruro de calcio se le hace más fácil captar las moléculas de agua haciéndolas más libres y fácil de eliminarlas. Esto asociado a la temperatura y la velocidad del flujo de aire (secador de bandejas) permiten la salida del agua a la superficie, lo que ocasiona una disminución de la velocidad de secado, teniendo como resultado un proceso de deshidratación más eficiente.

Existe una rápida remoción de humedad del producto, lo cual después esta remoción decreció dando lugar al incremento del tiempo de deshidratación. Este fenómeno muestra el efecto de la temperatura y velocidad del aire sobre el periodo de velocidad constante, donde se concluye que a mayor temperatura y velocidad de aire produceuna mayor remoción de agua no combinada, disminuyéndose los tiempos deeste periodo de secado. El mismo resultado fue obtenido en rebanadas de tomate (Doymaz, 2006) y (Akanbi, Adeyemi, y Ojo, 2006). Asimismo, como lo mencionado por Salcedo et al., 2014 después del período de velocidad constante existen dos fases en el período de velocidad decreciente; una primera fase en el cual el descenso de la humedad es parcialmente proporcional a la disminución de la velocidad de secado donde se remueve gran cantidad de agua y la segunda fase donde la velocidad de secado decrece debido a una lenta migración del agua al exterior del producto.

Las evaluaciones sensoriales realizadas con pruebas de aceptación por panelistas no entrenados, resultó en diferencias estadísticamente significativas en las características de color, textura y apariencia más no así en la característica olor de las muestras deshidratadas 
a un $95 \%$ de confianza. La mejor muestra con el mayor puntaje global fue: $\mathrm{T} 1\left(55^{\circ} \mathrm{C} ; 0,6 \mathrm{~m} / \mathrm{s}\right)$ con 3,25 puntos.

Se recomienda como otra investigación calcular los costos de este método de deshidratación, para poder industrializar este proceso y realizar el estudio de mercado así de este producto, ya que cuenta con un gran potencial en el mercado internacional, especialmente el mercado europeo España, Italia, entre otros y los países mediterráneos como Grecia, así lograr un potencial desarrollo del sector de productos deshidratados. Se recomienda también investigaciones comparando las otras variedades de tomate evaluando los parámetros físicoquímicos y el índice de madurez, de acuerdo a ello se puede tener un producto adecuado para diferentes mercados.

\section{REFERENCIAS BIBLIOGRÁFICAS}

Akanbi, C. T., Adeyemi, R. S., \& Ojo, A. (2006). Drying characteristics and sorption isotherm of tomato slices. Journal of Food Engineering 73, 141-146.

Badui Dergal, S. (2006). Química de los Alimentos. México: PEARSON EDUCACIÓN.

Barbosa-Cánovas, G. V., \& Vega-Mercado, H. (2000). Deshidratación de Alimentos. Zaragoza, España: ACRIBIA S.A.

Catraro, M. A. (19 de diciembre de 2014). Trabajo Final para optar por el grado académico: Especialista en Cultivos Intensivos. El Cultivo de la Higuera: Producción de higos y su deshidratación como método para el agregado de valor del producto. Esperanza, Santa Fe, Argentina: Facultad de Ciencias Agrarias. Universidad Nacional del Litoral.

Chang, C.-H., Lin, H.-Y., \& Liu, Y.-C. (2006). Comparisons on the antioxidant properties of fresh, freeze-dried and hot-air-dried tomatoes. Journal of Food Engineering 77, 478-485.

Claude, R. (2004). Reología y análisis de la textura de los alimentos.

Zaragoza: Editorial ACRIBIA S.A.

Cunningham, S. E., Mcminn, W. A., Magee, T. R., \& Richardson, P. S. (2008). Effect of processing conditions on the water absorption and texture kinetics of potato. Journal of Food Engineering, 214-223.

Doymaz, I. (2004). Sun drying of figs: an experimental study. Journal of Food Engineering 71, 403-407.

Doymaz, I. (2006). Air-drying characteristics of tomatoes. Journal of Food Engineering 78, 1291 - 1297.

Falade, K. O., \& Abbo, E. S. (2007). Air-drying and rehydration characteristics of date palm (Phoenix dactylifera L.) fruits. Journal of Food Engineering 49, 724-730.

FAO. (2007). Food and Agriculture Organizations of the United Nations. Obtenido de Food and Agriculture Organizations of the United Nations: www.fao.org

Garcia-Pascual, P., Sanjuán, N., Melis, R., \& Mulet, R. (2006). Morchella esculenta (morel) rehydration process modelling. Journal of Food Engineering 72 (4), 346-353.

Gómez Gómez, M. S. (diciembre de 2009). Tesis para obtener Título de Ingeniero en 
Alimentos. Deshidratado de tomate saladette en un secador de charolas giratorias. Huajuapan de León, Oaxaca, México.

Gómez, P. A., \& Camelo, A. F. (2002). Calidad postcosecha de tomates almacenados en atmósferas controladas. Horticultura Brasileira v. 20 (1), 38-43.

Guzmán Jara, V. J. (2008). Previo a la obtención del Título de: Ingeniería de Alimentos. Estudio de los Pre Tratamientos en las Características Físicas y Sensoriales del Tomate Deshidratado. Guayaquil, Ecuador: Escuela Superior Politécnica del Litoral.

Hardemburg, R. E., Watada, A., \& Wang, C. (1988). Almacenamiento comercial de frutas, legumbres y existencias de floristerías y viveros.

Hii, C., Jangam, S., Ong, S., \& Mujumdar, A. (2012). Solar Drying: Fundamentals, Applications and Innovations. Singapore.

Jenkins, J. (1948). The origin of the cultivated tomato. Economic Botany Vol.

2 Issue 4, 379-392.

Kerkhofs, N., Lister, C., \& Savage, G. (2005). Change in Colour and Antioxidant Content of Tomato Cultivars Following Forced-Air Drying. Plant Foods for Human Nutrition 60, 117-121.

Krokida, M. K., \& Marinos-Kouris, D. (2003). Rehydration kinetics of dehydrated products. Journal of Food Engineering 57, 1-7.

Maldonado, S., Arnau, E., \& Bertuzzi, M. A. (2009). Effect of temperature and pretreatment on water diffusion during rehydration of dehydrated mangoes. Journal of Food Engineering 96 (2010), 333 - 341.

Margaris, D. P., \& Ghiases, A. G. (2007). A study of hot air dehydration of sultana grapes. Journal of Food Engineering, 1115-1121.

Monsalve, J., \& Machado, M. (2007). Evaluación de dos métodos de deshidratación del tomate (Lycopersicom esculentum mill) variedad manzano. Multiciencias vol. 7 núm. 3, 256-265.

Moreno, D. C., \& Díaz, A. C. (s.f.). Influencia de las temperaturas del proceso de secado por aire caliente en las características de calidad de tomate (Lycopersicum esculentum).

Moreno, D. C., Sierra, H. M., \& Díaz-Moreno, C. (2014). EVALUACIÓN DE PARÁMETROS DE CALIDAD FÍSICO-QUÍMICA, MICROBIOLÓGICA Y SENSORIAL EN TOMATE DESHIDRATADO COMERCIAL

(Lycopersicum Esculentum). Revista U.D.C.A Actualidad \& Divulgación Científica 17

(1), 131-138.

ONU. (2007). Organización de las Naciones Unidas. Obtenido de Organización de las Naciones Unidas: www.un.org/es

Panzo, B. (4 de septiembre de 2012). Tesis para obtener el título de Licenciado en Biología. Caracterización físicoquímica ( $\mathrm{pH},{ }^{\circ}$ Brix, acidez y color) del tomate rojo (Lycopersicum esculentum mill) deshidratado y molido, como alternativa de manejo postcosecha. Peñuela, Veracruz, México: Facultad de Ciencias Biológicas y Agropecuarias.

Salcedo M, J., Mercado B., J. L., Vanegas B., M., Fernández, A. Q., \& Vertel M., M. L. (2014). Cinética de secado de la yuca (Manihot esculenta Crantz) variedad CORPOICA M-tai 
en función de la temperatura y de la velocidad de aire. Scielo 27 (2), 29-42.

Sanjuán, N., Simal, S., Bon, J., \& Mulet, A. (1999). Modelling of broccoli systems rehydration process. Journal of Food Engineering 42 (1), 27- 31.

Vázquez, T., Arnéz, S., Fernández, M., \& Fernández, J. (1997). Manual de secado solar técnico de alimentos. Cochabamba: ENERGETICA FAKT RC.

Yilmaz, E. (2001). The Chemistry of Fresh Tomato Flavor. Turkish Journal of Agriculture and Forestry 25, 149-155. 